\title{
(6) OPEN ACCESS \\ Reducing unnecessary urinary catheter use and other strategies to prevent catheter-associated urinary tract infection: an integrative review
}

\author{
Jennifer Meddings, ${ }^{1}$ Mary A M Rogers, ${ }^{1}$ Sarah L Krein, ${ }^{1,2}$ \\ Mohamad G Fakih, ${ }^{3}$ Russell N Olmsted, ${ }^{4}$ Sanjay Saint ${ }^{2,1}$
}

\section{- Additional material is published online only. To view please visit the journal online (http://dx.doi.org/10.1136/bmjqs- 2012-001774) \\ ${ }^{1}$ Department of Internal Medicine, University of Michigan Medical School, Ann Arbor, Michigan, USA \\ ${ }^{2}$ VA Ann Arbor Healthcare System, Ann Arbor, Michigan, USA \\ ${ }^{3}$ Department of Internal Medicine, St. John Hospital and Medical Center, Detroit, Michigan, USA \\ ${ }^{4}$ St. Joseph Mercy Health System, Ann Arbor, Michigan, USA}

\section{Correspondence to Dr Jennifer Meddings, Department of Internal Medicine, University of Michigan Medical School, 2800 Plymouth Road, Building 16, Room 427W Ann Arbor, MI 48109, USA; Meddings@umich.edu}

Received 19 December 2012 Revised 16 May 2013 Accepted 26 July 2013 Published Online First 27 September 2013

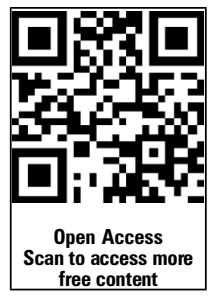

To cite: Meddings J, Rogers MAM, Krein SL, et al. BMJ Qual Saf 2014;23: 277-289.

\begin{abstract}
Background Catheter-associated urinary tract infections (CAUTI) are costly, common and often preventable by reducing unnecessary urinary catheter (UC) use.
\end{abstract}

Methods To summarise interventions to reduce UC use and CAUTIs, we updated a prior systematic review (through October 2012), and a meta-analysis regarding interventions prompting UC removal by reminders or stop orders.

A narrative review summarises other CAUTI prevention strategies including aseptic insertion, catheter maintenance, antimicrobial UCs, and bladder bundle implementation.

Results 30 studies were identified and summarised with interventions to prompt removal of UCs, with potential for inclusion in the meta-analyses. By meta-analysis (11 studies), the rate of CAUTI (episodes per 1000 catheterdays) was reduced by $53 \%$ (rate ratio $0.47 ; 95 \%$ $\mathrm{Cl} 0.30$ to $0.64, \mathrm{p}<0.001$ ) using a reminder or stop order, with five studies also including interventions to decrease initial UC placement. The pooled (nine studies) standardised mean difference (SMD) in catheterisation duration (days) was -1.06 overall ( $p=0.065)$ including a statistically significant decrease in stop-order studies (SMD -0.37; $p<0.001$ ) but not in reminder studies $(S M D,-1.54 ; p=0.071)$. No significant harm from catheter removal strategies is supported. Limited research is available regarding the impact of $U C$ insertion and maintenance technique. A recent randomised controlled trial indicates antimicrobial catheters provide no significant benefit in preventing symptomatic CAUTIs.

Conclusions UC reminders and stop orders appear to reduce CAUTI rates and should be used to improve patient safety. Several evidencebased guidelines have evaluated CAUTI preventive strategies as well as emerging evidence regarding intervention bundles. Implementation strategies are important because reducing UC use involves changing wellestablished habits.

\section{THE PROBLEM}

Urinary tract infection (UTI) has long been considered the most common healthcare-associated infection (HAI), with the vast majority of these infections occurring after placement of the convenient, uncomfortable, ${ }^{1}$ often unnecessary ${ }^{2-4}$ and easily forgotten urinary catheter. ${ }^{5}$ With an estimated $^{67} 449334$ healthcare-associated catheter-associated urinary tract infections (CAUTIs) per year, associated with an additional cost (in 2007) of US\$749-1007 ${ }^{7-9}$ per admission (or an estimated US $\$ 3744$ when complicated by blood stream infections), ${ }^{10}$ it is not surprising that CAUTIs were among the first hospital-acquired conditions selected for non-payment by Medicare as of October $2008,{ }^{11}$ and have been further targeted for complete elimination $^{12}$ as a 'never event,' with a national goal to reduce CAUTI by $25 \%$ by $2014 .^{13}$ These national initiatives renewed public and research interest in the prevention of CAUTI, prompting updates of several comprehensive guidelines ${ }^{14-17}$ and reviews of strategies to prevent CAUTI released since the 2001 Making Health Care Safer report. ${ }^{18}$

\section{WHAT STRATEGIES MAY PREVENT CATHETER-ASSOCIATED URINARY TRACT INFECTIONS?}

Similar to other HAIs - such as central line-associated blood stream infection many CAUTI prevention strategies have been 'bundled' into a composite of 


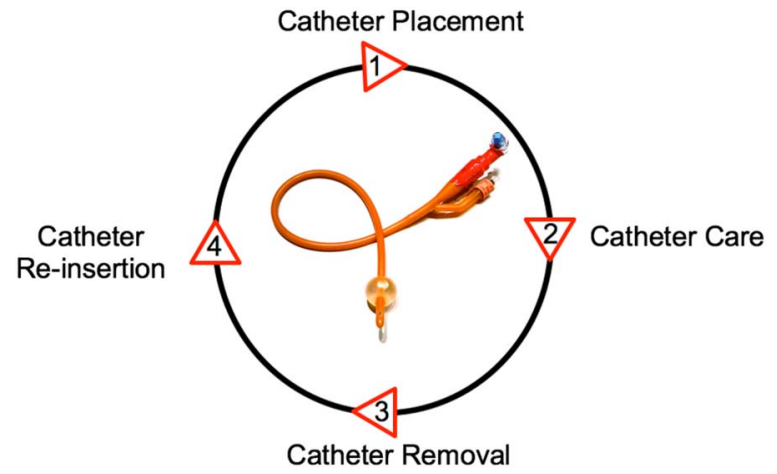

Figure 1 Lifecycle of the urinary catheter. ${ }^{21}$ This conceptual model illustrates four stages of the urinary catheter lifecycle as targets for interventions to decrease catheter use and catheter-associated urinary tract infection.

multimodal sets of interventions known as 'bladder bundles. ${ }^{19}$ These bundles consist of educational interventions to improve appropriate use and clinical skill in catheter placement, behavioural interventions such as catheter restriction and removal protocols, and use of specific technologies such as the bladder ultrasound. Despite some early success in implementing a bladder bundle ${ }^{19}$ to reduce urinary catheterisation rates, ${ }^{20}$ CAUTI prevention has proven challenging for several important reasons. For example, monitoring urinary catheter use and CAUTI rates to inform and sustain urinary catheter-related interventions is very resource intensive. Perhaps more importantly, improving practice regarding urinary catheter placement and removal also requires interventions to change the expectations and habits of nurses, physicians and patients about the need for urinary catheters.

To help organise and prioritise the many potential interventions to prevent CAUTI, we use the conceptual model of the 'lifecycle of the urinary catheter' highlight that the highest yield interventions to prevent CAUTI will target at least one of the four 'stages' of the catheter's 'life.' As illustrated in figure 1, the 'lifecycle' of the catheter (1) begins with its initial placement, (2) continues when it remains in place, day after day, (3) ceases when it is removed and (4) may start over if another catheter is inserted after removal of the first one.

Because avoiding unnecessary urinary catheter use is the most important strategy in prevention of CAUTI, we review the evidence on two types of interventions that target unnecessary urinary catheter use: (1) protocols and interventions to decrease unnecessary placement of urinary catheters (catheter lifecycle stage 1), and (2) interventions that prompt removal of unnecessary urinary catheters (catheter lifecycle stage 3 ).

\section{REVIEW PROCESS}

The evidence summarised in this chapter was generated using a literature search conducted for a prior systematic review and meta-analysis ${ }^{22}$ along with a focused update of the published peer-reviewed literature (from August 2008 to October 2012) through a MEDLINE search for intervention studies to reduce use of unnecessary urinary catheters in the acute care of adults. A CINAHL database search was also performed for interventions developed and implemented by nurses related to urinary catheter use. Studies were included if at least one outcome involving catheter use or CAUTI events (table 1) was reported as a result of the intervention, and with a comparison group (either preintervention vs postintervention, or a separate control group). Details of the systematic literature searches and methods employed for selection and reporting of the studies for this review are provided in the online supplementary appendix. The online supplementary appendix table summarises all the intervention studies described in this review, including study designs, patient populations and the interventions employed to avoid unnecessary catheter placement or to prompt catheter removal. This review was supported by the Agency for Healthcare Research and Quality (AHRQ), which had no role in the selection

Table 1 Description of outcomes evaluated (adapted from the prior meta-analysis ${ }^{22}$ )

Measures of Catheter-associated Urinary Tract Infection (CAUTI) development

Measures of urinary catheter use

Need for catheter replacement
Number of CAUTI episodes per 1000 catheter-days was recorded and a rate ratio was calculated to compare preintervention vs postintervention. When rates of both asymptomatic and symptomatic CAUTI were reported separately, ${ }^{57}$ the rates of symptomatic CAUTI were used for the meta-analysis. ${ }^{22}$ Cumulative risk of CAUTI during hospitalisation (ie, the percentage of patients who developed CAUTI) was also extracted for each study, and a risk ratio was calculated to compare risks before and after the intervention for the meta-analysis. ${ }^{22}$

Mean number of days of urinary catheter use per patient was recorded before and after the intervention, and a standardised mean difference was calculated to compare the two groups for the meta-analysis. ${ }^{22}$

Percentage of patient days in which the catheter was in place (reported by the study directly, or calculated from ratio of catheter days/patient days if provided in the study), for the comparison group (before intervention or control group) and postintervention

Percentage of patients studied who had a urinary catheter placed during the study period, for the comparison group (before intervention or control group) and postintervention

Recatheterisation need was extracted as the number and percent of patients who required replacement of a catheter after prior removal of an indwelling catheter. 
or review of the evidence or the decision to submit this manuscript for publication.

\section{WHAT STRATEGIES MAY REDUCE UNNECESSARY CATHETER USE?}

\section{Strategies to avoid unnecessary placement of indwelling} urinary catheters

Simply put, patients without urinary catheters do not develop CAUTI. Yet, multiple studies show that between $21 \%$ and $55.7 \% \%^{2420} 23-25$ of urinary catheters are placed in patients who do not have an appropriate indication and, therefore, may not even need a catheter. Over the past decade, several studies have employed interventions to decrease unnecessary catheter placement (described in the online supplementary appendix table). Although educational interventions are a common and important first step to decrease inappropriate catheter use, more effective and potentially more sustainable interventions go a step further by instituting restrictions on catheter placement. Protocols that restrict catheter placement can serve as a constant reminder for providers about the appropriate use of catheters, can suggest alternatives to indwelling catheter use (such as condom catheters or intermittent straight catheterisation), but perhaps most importantly, can generate accountability for placement of each individual urinary catheter. A fairly typical approach for developing a catheter restriction protocol is to begin with a basic list of appropriate catheter uses (such as the list provided in the Centers for Disease Control and Prevention's Healthcare Infection Control Practices Advisory Committee (HICPAC) guideline ${ }^{14}$ ); this list (see box 1) can then be tailored to include other indications based on local opinion and specialised patient populations.

The technology required to implement catheter placement restrictions ranges from low-technology strategies, such as a hospital or unit policy on appropriate catheter placement, or preprinted catheter orders with limited indications, to higher-technology strategies, such as computerised orders ${ }^{24}{ }^{26-28}$ for catheter placement. Catheter restriction protocols have been a common component of successful multimodal interventions to decrease catheter use and/or CAUTI rates, including hospital-wide ${ }^{24}$ interventions and interventions tailored for specific environments, such as the emergency department, ${ }^{23} 29$ inpatient units $^{2028}$ (including general medical ${ }^{26} 3031$ surgical $^{32}$ wards and $\left.\mathrm{ICU}^{32-36}\right)$, and in the periprocedural ${ }^{35}$ setting. Urinary retention protocols $s^{27} 31323^{35-37}$ are a type of catheter restriction protocols that often incorporate the use of a portable bladder ultrasound $^{27} 28313537-41$ to verify retention prior to catheterisation, and recommend use of intermittent catheterisation rather than indwelling catheters to manage a common and often temporary issue.
Box 1 Indications for indwelling urethral catheter use (from 2009 Centers for Disease Control and Prevention guideline ${ }^{14}$ )

A. Examples of appropriate indications for indwelling urethral catheter use

- Patient has acute urinary retention or bladder outlet obstruction

- Need for accurate measurements of urinary output in critically ill patients

- Perioperative use for selected surgical procedures:

1. Patients undergoing urologic or other surgery on contiguous structures of genitourinary tract

2. Anticipated prolonged surgery duration; catheters inserted for this reason should be removed in postanesthesia care unit

3. Patients anticipated to receive large-volume infusions or diuretics during surgery

4. Need for intraoperative monitoring of urinary output

- To assist in healing of open sacral or perineal wounds in incontinent patients

- Patient requires prolonged immobilisation (eg, potentially unstable thoracic or lumbar spine, multiple traumatic injuries such as pelvic fractures)

- To improve comfort for end-of-life care if needed

B. Examples of inappropriate uses of indwelling catheters

- As a substitute for nursing care of the patient or resident with incontinence

- As a means to obtain urine for culture or other diagnostic tests when patient can voluntarily void

- For prolonged postoperative duration without appropriate indications (eg, structural repair of urethra or contiguous structures, prolonged effect of epidural anesthesia, etc.)

\section{Strategies to prompt removal of unnecessary} urinary catheters

Urinary catheters are commonly left in place when no longer needed. ${ }^{4}{ }^{25}$ In most hospitals, four steps are required to remove a urinary catheter ${ }^{21}$ : (1) a physician recognises the catheter is in place, (2) the physician recognises the catheter is no longer needed, (3) the physician writes the order to remove the catheter and (4) a nurse removes the catheter. Thus, by default, hours and sometimes days may pass before an unnecessary catheter is recognised and removed. Because every additional day of urinary catheter use increases the patient's risk of infectious ${ }^{42}$ complications, and catheter use is also associated with non-infectious ${ }^{43}$ catheter-related complications, interventions that facilitate prompt removal of unnecessary catheters can have a strong impact. We describe below the evidence regarding strategies that may accelerate 
or bypass some of these four steps to prompt catheter removal.

Perhaps the most important CAUTI prevention strategy after placement of the catheter is to maintain awareness of the catheter's existence (in lifecycle stage 2 of figure 1), as healthcare providers may be unaware the catheter is in place. ${ }^{5}$ Thus, a key step in prompting removal of unnecessary catheters is frequently (by day or by shift) reminding nurses and physicians that the catheter remains in place. Catheter reminder interventions include a daily checklist ${ }^{24} 353644-46$ or verbal/ written reminder ${ }^{34}$ 47-51 to assess continued catheter need, a sticker reminder on the patient's chart ${ }^{38} 5253$ or catheter bag, ${ }^{54}$ or an electronic ${ }^{24}$ reminder that a catheter is still in place. Reminder interventions can be generated by nurses, physicians or electronic order sets, and can be targeted to remind either nurses or physicians about the catheter. Some reminder interventions have employed nurses dedicated to detecting unnecessary catheters. ${ }^{24} 38$ Reminder interventions can also serve to remind clinicians of appropriate catheter indications.

Unfortunately, reminder interventions can also be easy to ignore ${ }^{52}$ and catheters may remain in place without action. The next type of intervention to prompt removal of unnecessary catheters which goes a step further, is a 'stop order' that requires action. Stop orders prompt the clinician (either nurse or physician) to remove the catheter by default after a certain time period has elapsed or condition has occurred, unless the catheter remains clinically appropriate. For example, catheter stop orders can be configured to 'expire' in the same fashion as restraint or antibiotic orders, unless action is taken by a clinician. Stop orders directed at physicians ${ }^{24} \begin{array}{lllll}26 & 28 & 31 & 33 & 51\end{array}$ require an order to be renewed or discontinued on the basis of review at specific intervals, such as every $24-48 \mathrm{~h}$ after admission or postprocedure. Stop orders directed at nurses either require the nurse to obtain a catheter removal order from physicians, 303555 or can empower nurses to remove the catheter without requesting a physician order ${ }^{31} 33 \quad 3746 \quad 56-61$ on the basis of an appropriate indication list. Admittedly, implementing a nurse-empowered catheter removal protocol may be less effective than anticipated, as early qualitative research of nurse-empowered interventions indicate some nurses are uncomfortable with this autonomy ${ }^{59}$ and might not remove catheters as expected.

\section{BENEFITS AND HARMS}

What is the impact of strategies to avoid unnecessary urinary catheter use?

Impact of interventions to avoid unnecessary catheter placement Multiple before-and-after studies have found that interventions to decrease inappropriate catheter placement (such as catheter placement restrictions and urinary retention protocols) have resulted in a decrease in the use of urinary catheters, ${ }^{23} 242728313234364662$ a lower proportion of catheters in place without a physician order, 23242629 and a reduction in the proportion of catheters in place without an appropriate indication. ${ }^{23} 242931$

\section{Impact of reminder and stop-order interventions on catheter use and CAUTIs}

As an update to our prior systematic review and meta-analysis of 14 studies $^{22}$ published prior to August 2008, this systematic review (through October 2012) identified a total of 30 studies employing reminders and/or stop orders to prompt removal of unnecessary urinary catheters that reported at least one CAUTI or urinary catheter use measure defined in table 1. Table 2 summarises each of these 30 studies, including the outcomes reported, and a brief summary of the interventions employed (with more details regarding the interventions provided in the online supplementary appendix table). The majority (28) of studies were prepost designs, including three $^{32} 5052$ with concurrent controls; one study ${ }^{57}$ was a randomised control trial (RCT) and one study was a non-randomised crossover trial. ${ }^{26}$

With very similar results to the prior ${ }^{22}$ meta-analysis, the updated meta-analysis using 11 studies (figure 2, stratified by reminder vs stop order, and also see online supplementary appendix figure, stratified by study's focus on intensive care patients) indicated the rate of CAUTI (episodes per 1000 catheter-days) was reduced by $53 \%$ (rate ratio 0.47 ; $95 \%$ CI 0.30 to $0.64, \mathrm{p}<0.001$ ) with use of a reminder or stop order, with five of these studies also including interventions to restrict initial catheter placement. Based on this updated meta-analysis, reminders and stop orders could result in large numbers of avoided CAUTI episodes per 1000 catheter-days, particularly when baseline rates of CAUTI are high (table 3). Eight studies provided sufficient detail for pooling of the cumulative risk of CAUTI during the study period; the risk ratio for CAUTI was 0.72 (95\% CI 0.52 to $0.99 ; \mathrm{p}=0.045)$ for the intervention versus comparison groups (figure 3).

Using nine studies with sufficient detail for pooling (figure 4), the pooled standardised mean difference (SMD) in duration of catheterisation was -1.06 days overall $(p=0.065)$ including a statistically significant decrease in studies that used a stop order (SMD $-0.37 ; \mathrm{p}<0.001)$ but not in those that used a reminder (SMD, $-1.54 ; \mathrm{p}=0.071)$. Many studies reported other outcomes for urinary catheter use (detailed in table 2), but none of the studies identified since the prior meta-analysis provided sufficient detail (eg, number of patients in intervention or control groups, and measures of variability such as SD) to update the prior meta-analyses for these other urinary catheter use outcomes. 
Table 2 Summary of CAUTI and urinary catheter (UC) outcomes reported for studies with reminder or stop-order interventions, evaluated as potential studies to include in meta-analyses

\begin{tabular}{|c|c|c|c|c|c|c|}
\hline \multirow[b]{2}{*}{ 1st author (year) } & \multirow[b]{2}{*}{ Interventions* } & \multicolumn{2}{|l|}{ CAUTI outcomes } & \multicolumn{3}{|c|}{ Indwelling urinary catheter use outcomes } \\
\hline & & $\begin{array}{l}\text { CAUTI per } 1000 \\
\text { catheter days }\end{array}$ & $\begin{array}{l}\% \text { Patients who } \\
\text { developed CAUTI }\end{array}$ & $\begin{array}{l}\text { Mean days of UC } \\
\text { use per patient }\end{array}$ & $\begin{array}{l}\% \text { Patient days } \\
\text { with UC in use }\end{array}$ & $\begin{array}{l}\% \text { Patients with } \\
\text { UC used }\end{array}$ \\
\hline Adams $(2010)^{60}$ & $S$ & & & & & $25 \rightarrow 7.6$ \\
\hline $\begin{array}{l}\text { Andreessen } \\
(2012)^{28}\end{array}$ & $P, S, B$ & & & $5.6 \rightarrow 2.9 \dagger$ & & All catheterised $\neq$ \\
\hline $\begin{array}{l}\text { Apisarnthanarak } \\
(2007)^{47}\end{array}$ & $\mathrm{R}$ & $21.5 \rightarrow 5.2 \dagger$ & & $11.0 \rightarrow 3.0 \dagger$ & & $82 \rightarrow 84$ \\
\hline $\begin{array}{l}\text { Bruminhent } \\
(2010)^{53}\end{array}$ & $\mathrm{R}$ & $7.02 \rightarrow 2.08+\S$ & & & & All catheterised $\neq$ \\
\hline Cornia $(2003)^{26}$ & $P, S, E$ & & $8.8 \rightarrow 13.8$ & $8.0 \rightarrow 5.0 \dagger$ & & All catheterised $\ddagger$ \\
\hline Crouzet $(2007)^{48}$ & $\mathrm{R}$ & $12.3 \rightarrow 1.8 \dagger$ & $10.6 \rightarrow 1.1 \dagger$ & $8.4 \rightarrow 6.7$ & & All catheterised $\neq$ \\
\hline Dumigan $(1998)^{33}$ & $P, S, E$ & $14.3 \rightarrow 9.2$ & & & $74 \rightarrow 76$ & \\
\hline Elpern $(2009)^{34}$ & $P, R$ & $4.7 \rightarrow 0 \dagger$ & & & & All catheterised $\ddagger$ \\
\hline Fakih $(2008)^{50}$ & $\mathrm{R}$ & & & & $20 \rightarrow 16 \dagger$ & \\
\hline Fuchs $(2011)^{35}$ & $P, S$ & $2.88 \rightarrow 1.46$ & & & & All catheterised $\ddagger$ \\
\hline Gotelli $(2008)^{56}$ & S & & & & & $24.0 \rightarrow 17.0$ \\
\hline Huang $(2004)^{49}$ & R & $11.5 \rightarrow 8.3 \dagger$ & $3.8 \rightarrow 2.4 \dagger$ & $7.0 \rightarrow 4.6 \dagger$ & & $74.5 \rightarrow 76.2$ \\
\hline Jain $(2006)^{44}$ & $R, B, A$ & $3.8 \rightarrow 2.4$ & & & & \\
\hline Knoll $(2011)^{24}$ & $P, R, S, B, O$ & & & & Mean daily UC pres & lence: $15.2 \rightarrow 13.7 \S$ \\
\hline Loeb $(2008)^{57}$ & S & & $2.1 \rightarrow 2.1$ & $5.0 \rightarrow 3.7 \dagger$ & & All catheterised $\neq$ \\
\hline Murphy $(2007)^{54}$ & $\mathrm{R}, \mathrm{E}$ & Reduced UTI rates & $30 \%$, no details & & & \\
\hline Reilly $(2008)^{36}$ & $P, R, E$ & & & $4.7 \rightarrow 3.0$ & $96 \rightarrow 86$ & All catheterised $\neq$ \\
\hline Robinson $(2007)^{55}$ & $S$ & & $40.0 \rightarrow 13.3$ & $8.6 \rightarrow 4.5$ & & \\
\hline Roser $(2012)^{61}$ & $P, S, O$ & CAUTI (details uncl & r): $45 \rightarrow 28$ & & $\begin{array}{l}\text { UC 'Utilisation rate } \\
\text { (not further define }\end{array}$ & $\begin{array}{l}: \text { ICU: } 84 \rightarrow 68 \\
\text { MedSurg: } 16 \rightarrow 11\end{array}$ \\
\hline Rothfield $(2010)^{30}$ & $P, S$ & $3.2 \rightarrow 2.4$ & & & $33.3 \rightarrow 18.5 \dagger$ & $33.3 \rightarrow 18.8$ \\
\hline Saint $(2005)^{52}$ & $\mathrm{R}$ & & & $0.6 \rightarrow 0.4 \dagger$ & $14 \rightarrow 13$ & \\
\hline Schultz $(2011)^{37}$ & $P, S$ & & & & $90-95 \rightarrow 76-84$ & \\
\hline Sequin $(2010)^{51}$ & $S$ & $5.0 \rightarrow 4.9$ & $4.3 \rightarrow 3.0$ & Median $5.0 \rightarrow 4.0 \dagger$ & & $92.7 \rightarrow 91.2$ \\
\hline Stephan $(2006)^{32}$ & $P, S, E$ & $45.8 \rightarrow 18.6 \dagger$ & & $5.0 \rightarrow 3.9 \dagger$ & & \\
\hline Titsworth $(2012)^{46}$ & $P, R, S, B, A, 0$ & $13.3 \rightarrow 4.0 \dagger$ & & & $\begin{array}{l}\text { UC Utilisation rate } \\
\text { UCs: } 100 \rightarrow 73\end{array}$ & of ICU beds with \\
\hline Topal $(2005)^{31}$ & $P, S, E$ & $36.0 \rightarrow 19.0 \dagger$ & $19.5 \rightarrow 12.3$ & & $16 \rightarrow 10 \dagger$ & \\
\hline $\begin{array}{l}\text { Van den Broek } \\
(2011)^{38}\end{array}$ & $P, R, S, O$ & & $12.6 \rightarrow 12.7$ & $\begin{array}{l}\text { ICU/CCU: } 25.6 \rightarrow 16.2 \\
\text { Med: } 14.9 \rightarrow 12.5 \dagger \\
\text { Neuro: } 15.8 \rightarrow 15.7 \\
\text { Surg: } 11.8 \rightarrow 10.5 \dagger\end{array}$ & & $\begin{array}{l}\text { ICU/CCU: } 74 \rightarrow 81 \\
\text { Med: } 14 \rightarrow 14 \\
\text { Neuro: } 20 \rightarrow 19 \\
\text { Surg: } 21 \rightarrow 19 \dagger\end{array}$ \\
\hline Voss $(2009)^{58}$ & $S$ & & & $4.9 \rightarrow 3.9$ & $26 \rightarrow 10$ & $33 \rightarrow 15.3$ \\
\hline Weitzel $(2008)^{45}$ & $\mathrm{R}$ & & $37 \rightarrow 6.7$ & $8.6 \rightarrow 4.5$ & & \\
\hline Wenger $(2010)^{59}$ & $S, E, A$ & $2.26 \rightarrow 1.02 \dagger$ & & & & \\
\hline
\end{tabular}

A grey-shaded table cell indicates that the outcome was not reported in the study.

*Intervention key (note: interventions described in more detail in online supplementary appendix table).

tDifference of $p<0.05$ reported between comparison group (before intervention or control) $\rightarrow$ postintervention group.

$\ddagger$ Not applicable, as only catheterised patients were included for these studies (so 100\% catheterised).

§Reporting first published postintervention result, for this study that reported serial postintervention results.

$\mathrm{P}=$ Intervention to avoid unnecessary catheter Placement (eg, restricted indications of use, bladder scanner protocols, etc).

$\mathrm{R}=$ Reminder intervention that $\mathrm{UC}$ is still present, could be directed at either physician or nurse.

$S=\bar{S}$ top order intervention, prompting/requiring removal of the UC based on time or clinical criteria.

$\mathrm{E}=\overline{\mathrm{E}}$ ducation regarding UC placement or maintenance.

$\mathrm{B}=\overline{\mathrm{B}}$ ladder bundle of interventions (which included UC placement, maintenance care standardisation).

$A=\bar{A}$ ntimicrobial UCs.

$0=\overline{0}$ ther CAUTI or catheter-related interventions, detailed in online supplementary appendix table.

CAUTI, catheter-associated urinary tract infection; CCU, coronary care units; ICU, intensive care units; UTI, urinary tract infection. 


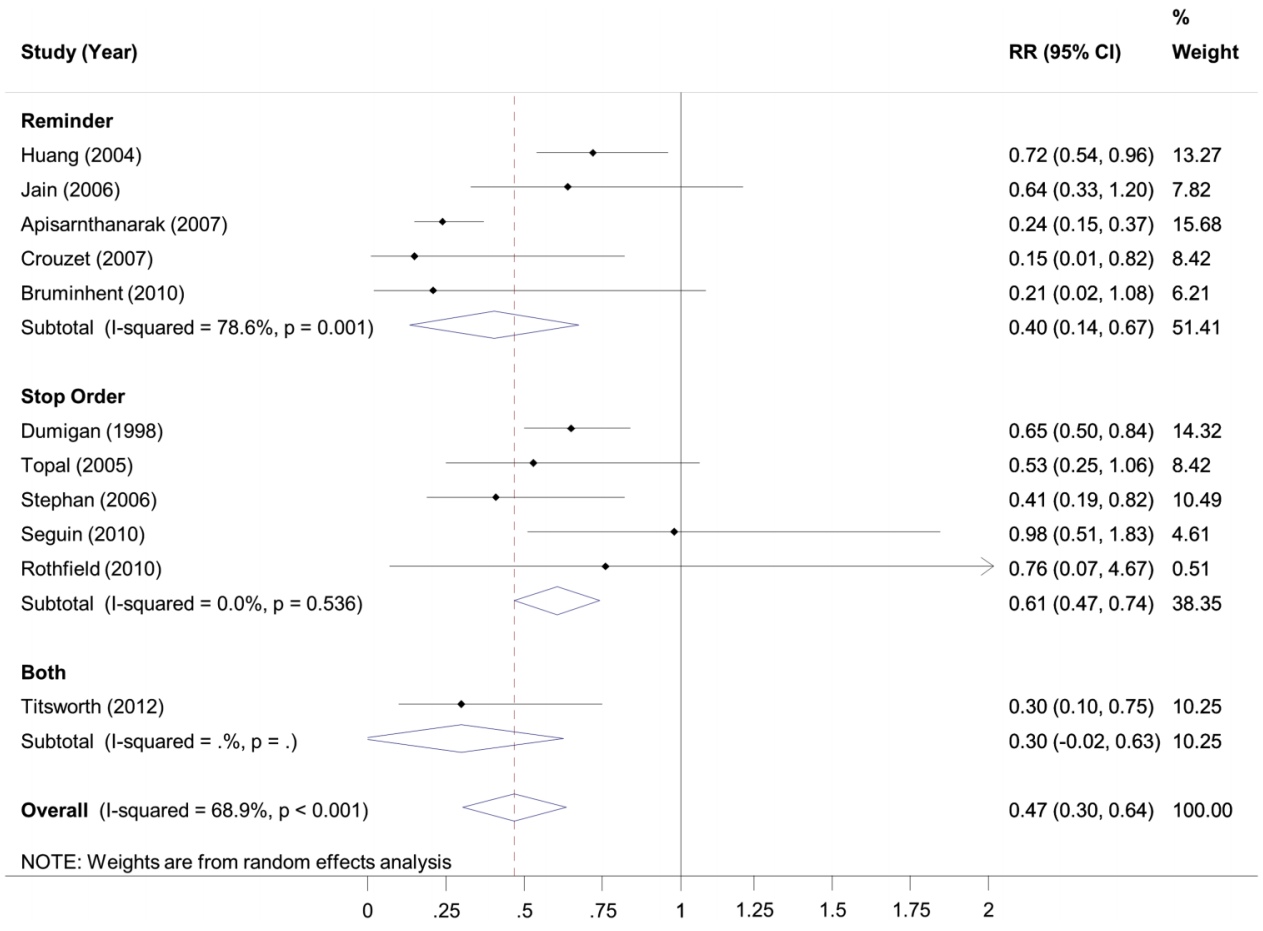

Figure 2 Meta-analysis of rate ratios for catheter-associated urinary tract infection episodes per 1000 catheter days, for intervention versus control groups, stratified by type of intervention to prompt catheter removal.

The one $\mathrm{RCT}^{57}$ that used a urinary catheter stop order warrants further discussion. In this study focusing on catheterised general medicine patients, urinary catheter use decreased more in the 347 patients in the stop-order group compared to 345 patients receiving usual care by -1.34 days $(95 \%$ CI -0.64 to -2.05 days, $\mathrm{p}<0.001)$. Unexpectedly, symptomatic CAUTI rates did not change, occurring in $2.1 \%$ of both stop-order and usual-care groups $(p=0.99)$. Of note, Loeb et al did not report CAUTIs per 1000 catheter days, so these results could not be included in pooled rate ratios (figure 2), but were included in pooled risk ratios (figure 3). There are several potential reasons why a decrease in CAUTIs was not seen in this study, including high rates of antimicrobial use in both controls and stop-order groups (protective against CAUTI, and a variable not often reported in these comparative studies), and the possibility that a

Table 3 Number of avoided CAUTI episodes per 1000 catheter days

\begin{tabular}{|c|c|c|c|}
\hline \multirow{2}{*}{$\begin{array}{l}\text { Baseline rate of } \\
\text { CAUTI episodes } \\
\text { per } 1000 \text { catheter } \\
\text { days }\end{array}$} & \multicolumn{3}{|c|}{$\begin{array}{l}\text { Number of avoided CAUTI episodes per } \\
1000 \text { catheter days anticipated by the type } \\
\text { of intervention to prompt catheter removal }\end{array}$} \\
\hline & Reminder & $\begin{array}{l}\text { Stop } \\
\text { order }\end{array}$ & Overall \\
\hline 5 & 3.0 & 2.0 & $2.7(95 \% \mathrm{Cl} 1.8$ to 3.5$)$ \\
\hline 10 & 6.0 & 4.0 & $5.3(95 \% \mathrm{Cl} 3.6$ to 7.0$)$ \\
\hline 20 & 11.9 & 7.9 & $10.6(95 \% \mathrm{Cl} 7.3$ to 13.9$)$ \\
\hline
\end{tabular}

CAUTI, catheter-associated urinary tract infections. 1-day reduction in catheter use may not be sufficient to demonstrate an effect on the risk of UTI, although the effects on rates of UTI (by tracking catheter days) are not known.

Potential for unintended harm by catheter removal interventions Interventions that facilitate removal of urinary catheters pose the risk of premature urinary catheter removal, with patients then requiring unnecessary recatheterisation; any catheterisation event is associated with procedure-related discomfort and other potential complications. Thus, monitoring the need for recatheterisation is important to avoid unintended patient harm. In the meta-analysis of reminder and stop-order studies, only four of the 14 studies reported rates of recatheterisation ${ }^{26} 485257$ with low recatheterisation rates noted in both intervention and control groups. None of the 16 more recent studies involving reminders or stop orders to prompt catheter removal reported data on potential patient harm, such as premature removal.

\section{SUMMARY OF OTHER STRATEGIES TO PREVENT CAUTI}

Several recent evidence-based guidelines ${ }^{14-17}$ have focused on preventing CAUTI, and have assessed the evidence and provided recommendations for implementing prevention strategies. Key recommendations in the Centers for Disease Control and Prevention (CDC) guideline ${ }^{14}$ in addition to appropriate catheter use (box 1), include (1) aseptic insertion of urinary catheters by properly trained personnel, using aseptic 


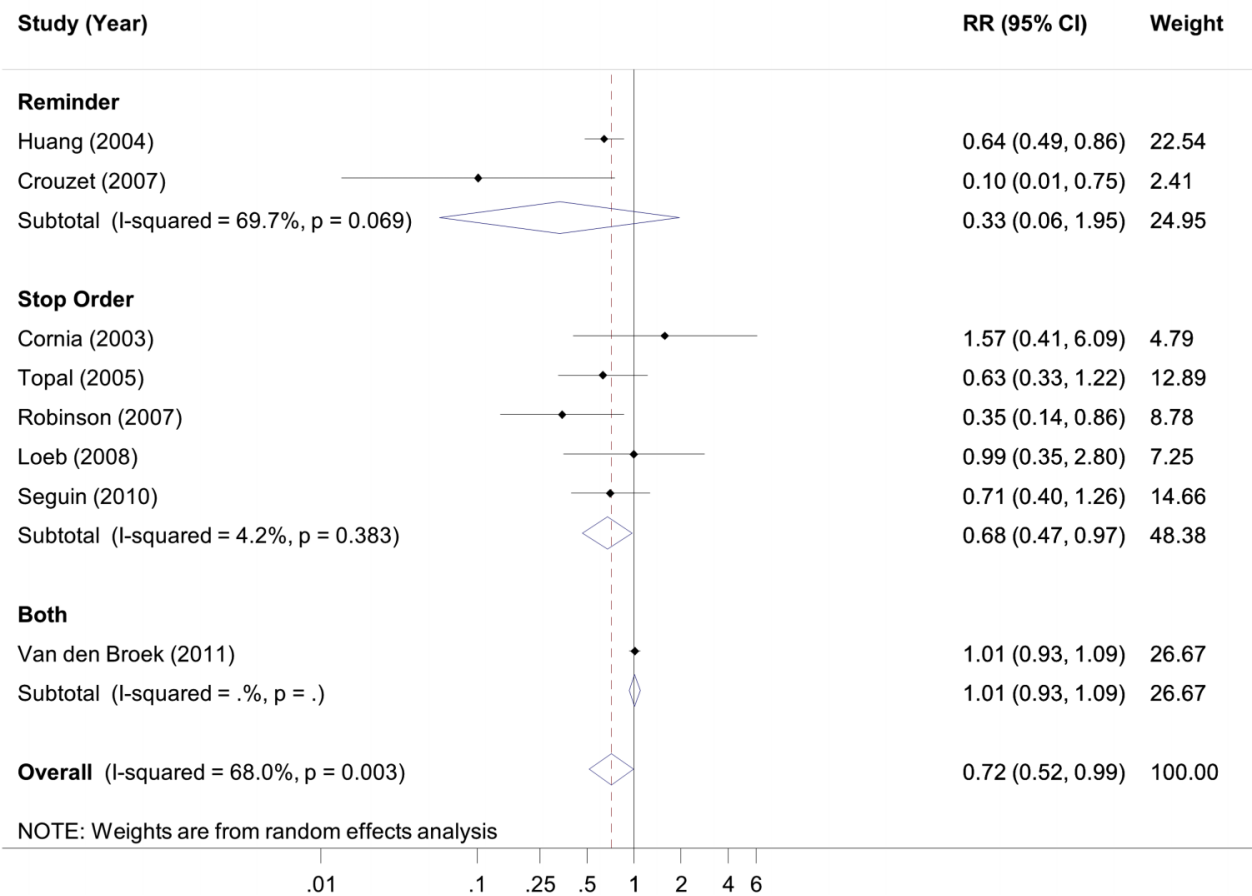

Figure 3 Meta-analysis of risk ratios for percentage of patients who developed catheter-associated urinary tract infection, for intervention versus control groups, stratified by type of intervention to prompt catheter removal.

technique and sterile equipment (with an exception being that clean technique is appropriate for chronic intermittent catheterisation) and (2) proper urinary catheter maintenance with a sterile, closed drainage system permitting unobstructed urine flow. Aseptic insertion is primarily recommended as a standard of care for which limited evidence exists. Stronger evidence (epidemiological and clinical) supports the

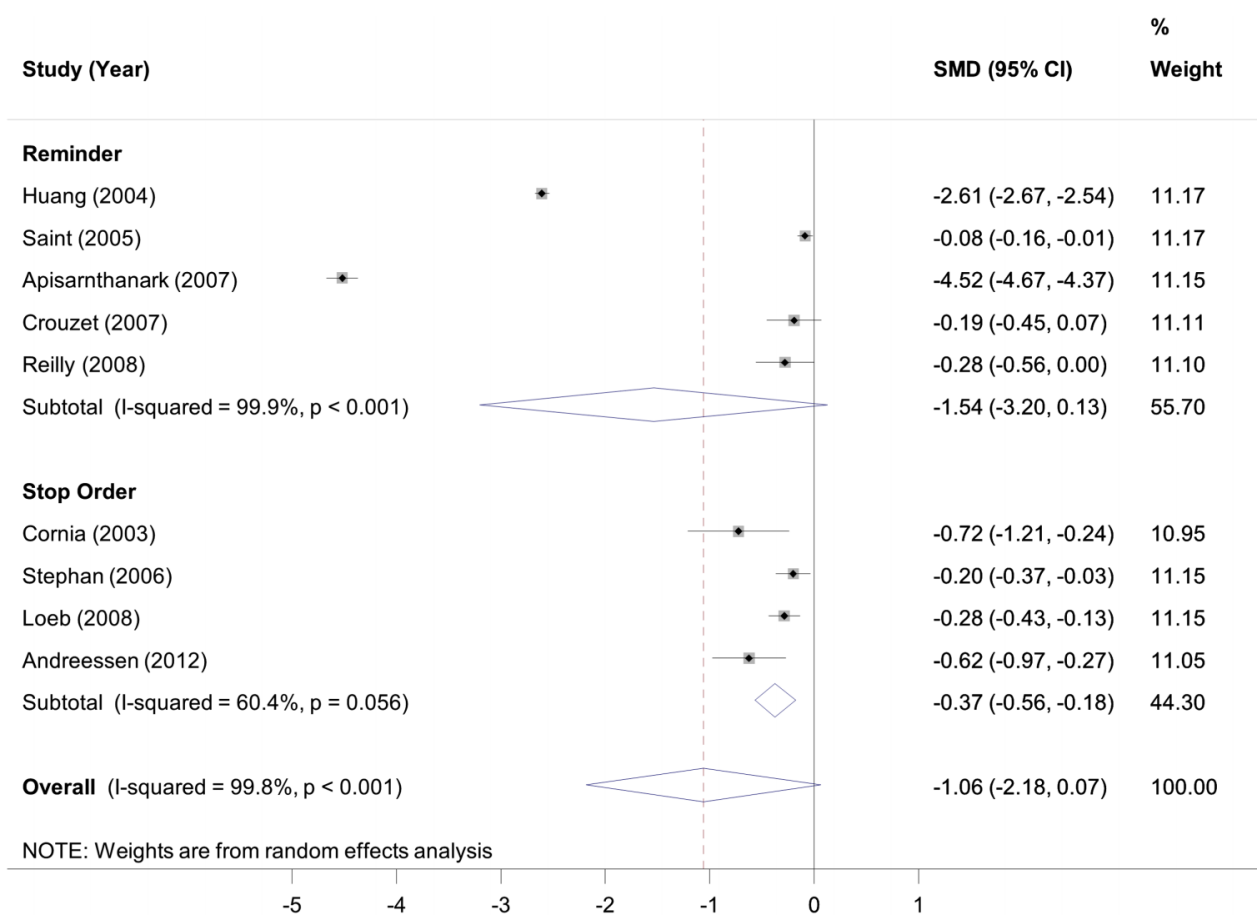

Figure 4 Meta-analysis of the standardised mean difference in days of urinary catheter use, for intervention versus control groups, stratified by type of intervention to prompt catheter removal. 
importance of a sterile, closed, unobstructed urinary drainage system.

A more controversial topic has been the use of antimicrobial catheters. Based on evidence available before 2010, the most recent CDC guideline recommended $^{14}$ that antimicrobial catheters should not be used routinely to prevent CAUTI. It suggested that further research was needed both on the effect of silver-alloy-coated catheters in reducing the risk of clinically significant CAUTI outcomes, and on the benefit of silver-alloy-coated catheters in selected patients at high risk of infection. In November 2012, a long-awaited multicentre RCT demonstrated a lack of effectiveness of antimicrobial catheters (including silver-alloy and nitrofurazone-releasing catheters) to provide significant and clinically important reductions in symptomatic CAUTIs. ${ }^{63}$

Bundles of interventions are also an important strategy, as part of a multimodal approach that focuses efforts on high-yield interventions. For example, one strategy that includes several of the components from the bladder bundle implemented by the Michigan Health and Hospital Association (MHA) Keystone Center for Patient Safety \& Quality is the 'ABCDE' approach $^{19}$ :

- Adherence to general infection control principles is important (eg, hand hygiene, surveillance and feedback, aseptic insertion, proper maintenance, education).

- Bladder ultrasound may avoid indwelling catheterisation.

- Condom catheters or other alternatives to an indwelling catheter such as intermittent catheterisation should be considered in appropriate patients.

- Do not use the indwelling catheter unless you must!

- Early removal of the catheter using a reminder or nurse-initiated removal protocol appears warranted.

Several studies ${ }^{24} \quad 28 \quad 44 \quad 46$ have included bladder bundles as interventions for decreasing catheter use and/or CAUTI rates, including a statewide initiative ${ }^{20}$ leading to significant increases in the proportion of catheters used for appropriate indications (from $44.3 \%$ to $57.6 \%, \mathrm{p}=0.005$ ).

\section{IMPLEMENTATION CONSIDERATIONS}

What methods have been used to improve the

implementation of interventions to prevent

catheter-associated urinary tract infections?

Because reducing unnecessary catheter use often requires changing well-established habits and beliefs of nurses and physicians, the challenge of implementation should not be underestimated. To facilitate implementation of practices to prevent CAUTI, the Michigan Keystone Bladder Bundle Initiative ${ }^{19} 20$ used the Johns Hopkins University collaborative model for transformational change. This model is based, in part, on the 'four Es': Engage, Educate, Execute, Evaluate. ${ }^{64}$ During the 'Engage' and 'Educate' steps, hospitals were provided information in multiple formats and a toolkit describing the intervention steps and outcomes measures. In the 'Execute' step, the hospital was strongly encouraged to choose one nurse champion ${ }^{50}{ }^{65}$ (eg, a case manager, nurse coordinator, or clinical nurse specialist) to lead the initiative and organise a bladder bundle team, including at least one physician, and to participate in workshops and conference calls with other participating hospitals to provide additional expert content and practical coaching. Also during the 'Execute' step, daily patient rounds (which in some hospitals were called a 'catheter patrol') were recommended to assess catheter presence and necessity, and provide feedback to specific units and re-evaluate strategies in progress. Hospitals were also encouraged to implement more active strategies for prevention, such as a catheter reminder system or promoting the use of catheter alternatives by developing protocols or making sure the necessary supplies were readily available. In the 'Evaluate' phase, hospitals were asked to assess improvements in catheter use and appropriateness according to specified indications and to address any barriers to progress and sustainability.

Implementation challenges within CAUTI prevention should be expected ${ }^{66}$ and managed accordingly. Qualitative assessment focusing on HAI prevention has identified two important potential barriers to HAI preventive efforts: 'active resisters' and 'organisational constipators. ${ }^{67}$ Active resisters are hospital personnel who vigorously and openly oppose changes in practice, as a matter of habit or culture (eg, 'just not how they were trained'). Management of active resisters often requires those in authority to mandate compliance, collect data and provide feedback. ${ }^{67} \mathrm{~A}$ 'champion' who is influential, or a peer of the resisting staff, may also help to overcome active resistance. ${ }^{65} 66$ 'Organisational constipators' are usually mid-level or high-level executives who act as barriers to change by preventing or delaying certain actions needed to implement new practices. ${ }^{67}$ Strategies to address an organisational constipator are to include this person in early discussions to improve buy-in and motivation, working around the person, or replacing the constipator.

A unique challenge to expect when implementing urinary catheter removal strategies is reluctance by some nurses to remove the catheter, ${ }^{66}$ even when the nurse is 'empowered' to do so. In some cases, nurses may be active resisters due to disagreement with the catheter policy and/or a desire to avoid the inconveniences and increased frequency of patient contact required for the care of incontinence and catheter alternatives. There is also a competing pressure on nurses to mitigate development of hospital-acquired pressure ulcers-leading to perception that urinary catheters could lessen risk of skin breakdown despite being only indicated ${ }^{14}$ for patients with incontinence and open sacral wounds. Other nurses report they simply do not feel comfortable $^{59}$ removing the 
catheter without explicit orders from the physician, which is ironic considering that many nurses place catheters without orders. Nursing comfort with catheter removal can be increased ${ }^{59}$ with peer support and education, and may be facilitated by directly addressing the workload concerns associated with the removal of indwelling catheters. Indeed, a survey of nurses ${ }^{30}$ during implementation of a nurse-empowered catheter removal protocol indicated increased nursing and patient satisfaction, despite the expected increase in workload. Recent successful and sustainable multimodal interventions to decrease catheter use including nurse-driven efforts had bedside nurses who, by survey, ${ }^{62}$ viewed themselves as responsible for evaluation of catheter presence and need, had confidence in their knowledge, and viewed physicians as receptive to their requests regarding catheter removal.

Even though CAUTI is a very common HAI, Krein and colleagues reported that CAUTI preventive practice use is lagging behind efforts to prevent central line-associated bloodstream infection and ventilatorassociated pneumonia, ${ }^{68}$ with room for improvement in adopting catheter removal and CAUTI preventive strategies demonstrated again in two recent large surveys of hospitals ${ }^{69}$ and ICUs. ${ }^{70}$ Fortunately, many resources exist (http://www.catheterout.org) to help hospitals develop and implement programmes to decrease catheter use and prevent CAUTI, including a range of tools and educational materials to address implementation challenges. Hospital and unit-level leadership also play a key role in preventing infection. $^{71}$

\section{Monitoring and providing feedback on catheter use and CAUTI rates}

Inappropriate urinary catheter use is an easy habit to start and a difficult one to break. ${ }^{21}$ Consequently, many studies ${ }^{20} 33$ have emphasised the importance of on-going surveillance and feedback as an intervention to reduce HAIs, such as CAUTI, and sustain ${ }^{20} 2462$ prevention efforts. New national efforts to reduce CAUTI (http://www.onthecuspstophai.org/stop-cauti/) incorporate periodic feedback to participating units on urinary catheter use and CAUTI rates. The CAUTI rates evaluated include the National Healthcare Safety Network (NHSN) and the newly described population-based rates. $^{72}$ The population-based CAUTI rate incorporates both the NHSN rate and the device utilization ratio, to account for interventions focused on reduction in catheter use and improvements in placement and maintenance.

Important next steps to address CAUTI involve developing strategies to decrease the effort, and resources required to monitor catheter use and CAUTI rates. Advanced informatics tools have recently been shown to increase the impact of this feedback loop to the extent that rates of CAUTI were lower in facilities that deployed these tools compared to those that did not. ${ }^{73}$ Careful selection or development of datasets used for implementing hospital payment changes and public reporting for CAUTI events is also recommended. Unfortunately, the current administrative data used to implement nonpayment ${ }^{11}$ for hospital-acquired CAUTIs, and to publicly report hospital performance, likely captures few CAUTI events, given documentation and coding challenges $^{74}$ to translate a UTI event from a medical record into hospital-acquired CAUTI in the administrative datasets.

\section{What is the cost of implementing a CAUTI prevention program?}

The cost of implementing a CAUTI prevention programme will vary based on the level of technology used (eg, computerised vs preprinted catheter orders, and whether portable bladder ultrasounds are purchased) and the time invested in implementing and evaluating the interventions. Saint and colleagues, in their study of a written urinary catheter reminder generated by a research nurse to remind physicians which of their inpatients had urinary catheters, ${ }^{52}$ found that the intervention was either cost-neutral or modestly cost-saving depending on the assumptions made. More recently, a study ${ }^{38}$ of five hospitals in The Netherlands employed a multimodal intervention including reminders in four hospitals, and a stop order in the fifth hospital. The programme was found to be cost saving, with the mean amount saved being $€ 537$ (or US\$700) per 100 hospitalised patients.

\section{DISCUSSION}

In summary, healthcare providers should strongly consider employing interventions to avoid unnecessary catheter placement (such as catheter placement restrictions) and to prompt removal of unnecessary catheters by reminders and stop orders, with special consideration for nurse-initiated removal protocols. The strength of the evidence is moderate to high. These interventions appear to be low cost, low risk, effective and sustainable 202462 strategies to address a common hospital-acquired infection in the USA, with some unique but not impossible challenges for implementation. Moreover, UTIs are increasingly caused by multidrug-resistant organisms (MDROs) including strains that are resistant to all available therapeutic agents; ${ }^{75} 76$ preventing CAUTI may help patients avoid MDRO infection.

Identifying and supporting an appropriate 'champion' who is influential among staff can be crucial to help overcome resistance to change behaviour regarding catheter use and facilitate the adoption of preventive strategies. Monitoring and providing feedback of catheter use and CAUTI rates is important in the implementation and continued use of CAUTI preventive strategies. Furthermore, reducing indwelling catheter use addresses the non-infectious complications of 
urinary catheter use such as catheter-related patient discomfort and immobility. Unfortunately, hospitals' surveys have repeatedly demonstrated low and only slowly improving adoption of CAUTI preventive strategies, such as reminders, stop orders and condom catheters compared to higher rates of adoption of antimicrobial urinary catheters in non-federal hospitals. ${ }^{68}$ Yet, the negative results of a recent randomised controlled trial on the effectiveness of antimicrobial urinary catheters ${ }^{63}$ is expected to translate into reduced use of these catheters.

Interest and resources for preventing CAUTI is likely to grow due to expanding national initiatives focused on reducing hospital-acquired infections, including CAUTI, being implemented with a combination of mandated public reporting and reduced pay to hospitals. Current prevention efforts include a 50-state initiative-'On the CUSP: Stop CAUTI'- that is funded by the AHRQ and led by the Health Research and Educational Trust (HRET) of the

\section{Key summary points}

- Multiple before-and-after studies have found that interventions to decrease inappropriate catheter placement (such as catheter placement restrictions or urinary retention protocols) have resulted in decreased catheter use, fewer catheters in place without physician orders, and fewer catheters in place without an appropriate indication.

- An updated systematic review and meta-analysis found that catheter reminders or stop orders decreased catheter-associated urinary tract infections (CAUTIs) by $53 \%$. An updated literature review identified many recent interventions with reminders or stop orders reducing CAUTI rates and/or urinary catheter use.

- Bundles of interventions focusing on strategies to reduce unnecessary catheter use (by avoiding unnecessary placement and prompting removal) and decrease CAUTI rates by general infection control principles (hand hygiene, surveillance/feedback, aseptic insertion, etc) have been successful, particularly when coupled with implementation strategies to address common barriers encountered when trying to change well-established habits of physicians and nurses.

- The role of antimicrobial catheters in preventing CAUTI has been addressed by a recent large randomised controlled trial showing no significant additional benefit in reducing symptomatic CAUTIs.

- CAUTI prevention costs vary by programme, but such programmes have been demonstrated to be cost saving.
American Hospital Association. AHRQ has made available a toolkit for CAUTI prevention (http://www. onthecuspstophai.org/on-the-cuspstop-cauti/

toolkits-and-resources/). This initiative is providing resources and implementation expertise to hospitals and state organisations that are beginning CAUTI prevention programmes, with a goal of reducing CAUTI rates by $25 \%$ by focusing on high-yield interventions such as those reducing urinary catheter use.

Acknowledgements We thank Andrew Hickner, MSI, for conducting the update of the literature search and for providing assistance with references and manuscript editing, and Whitney Townsend, MLIS, for performing the literature search for the original $^{22}$ systematic review.

Contributors JM and SS designed the systematic review, conceived the manuscript, and assembled the authorship team. JM performed the systematic review and abstracted the data required for the review overall (as summarised in text and tables) and the meta-analysis, and wrote the first draft of the manuscript. JM and MAMR had full access to the data reviewed and analysed for this study; MAMR conducted the meta-analysis, and interpreted the results. JM and MAMR take responsibility for the integrity and accuracy of the data for the meta-analysis; JM (the guarantor) also takes responsibility for the integrity and accuracy of all data summarised in the manuscript. MAMR and SS also reviewed and revised the manuscript critically for important intellectual content. SLK, MGF, and RNO also contributed substantially to design of the manuscript, and reviewed and edited the manuscript for important intellectual content. All authors approved the final version to be published.

Funding This work was supported by funding from the Agency for Healthcare Research and Quality (AHRQ), US Department of Health and Human Services (Contract No.

HHSA-290-2007-10062I). AHRQ reviewed contract deliverables to ensure adherence to contract requirements and quality, and a copyright release was obtained from AHRQ prior to submission of this manuscript.

Competing interests Some of the authors have also received grant support from the Agency for Healthcare Research and Quality (AHRQ Award 1R010HS018344-01A1 to JM, MAMR and SS, and Award 1K08-HS019767-01 to JM) and the

National Institute of Diabetes and Digestive and Kidney Diseases (Award R21-DK078717, to SS), and the National Institute of Nursing Research (Award R01-NR010700, to SLK and SS). JM is a recipient of the National Institutes of Health Clinical Loan Repayment Program for 2009 to 2013. Authors have also received support for the development and implementation of the 'On the CUSP: Stop CAUTI' Initiative serving as members of the National Leadership Team (SK, MGF, SS), technical expert panel (RNO), and core national faculty (JM, RNO, MAMR), through a subcontract with Health Research and Educational Trust through AHRQ. MGF also receives support from the Ascension Health Hospital Engagement Network effort to prevent healthcare-associated infections and Michigan Health \& Hospital Association Hospital Engagement Network to reduce CAUTI. RNO is also a member of speakers' bureau for Ethicon, Inc., Sage Products and Avid Education Partners, LLC; and serves as a consultant to Premier Inc, and previously served as a consultant for Bard Medical. SS has received numerous honoraria and speaking fees from academic medical centres, hospitals, specialty societies, state-based hospital associations, group-purchasing organisations (eg, VHA, Premier), and non-profit foundations (eg, Michigan Health and Hospital Association, Institute for Healthcare Improvement) for lectures about catheter-associated urinary tract infection and implementation science. JM has received numerous honoraria from academic medical centers, hospitals, specialty societies and state-based hospital associations for lectures related to catheter-associated urinary tract infection. Author(s) declare no competing financial 
interests exist. All statements expressed in this work are those of the authors and should not in any way be construed as official opinions or positions of the RAND Corporation, US Department of Veterans Affairs, AHRQ, or the US Department of Health and Human Services.

Provenance and peer review Not commissioned; externally peer reviewed.

Open access This is an Open Access article distributed in accordance with the Creative Commons Attribution Non Commercial (CC BY-NC 3.0) license, which permits others to distribute, remix, adapt, build upon this work noncommercially, and license their derivative works on different terms, provided the original work is properly cited and the use is non-commercial. See: http://creativecommons.org/licenses/ by-nc/3.0/

\section{REFERENCES}

1 Saint S, Lipsky BA, Baker PD, et al. Urinary catheters: what type do men and their nurses prefer? J Am Geriatr Soc 1999;47:1453-7.

2 Gokula RR, Hickner JA, Smith MA. Inappropriate use of urinary catheters in elderly patients at a midwestern community teaching hospital. Am J Infect Control 2004;32:196-9.

3 Fakih MG, Shemes SP, Pena ME, et al. Urinary catheters in the emergency department: very elderly women are at high risk for unnecessary utilization. Am J Infect Control 2010;38:683-8.

4 Jain P, Parada JP, David A, et al. Overuse of the indwelling urinary tract catheter in hospitalized medical patients. Arch Intern Med 1995;155:1425-9.

5 Saint S, Wiese J, Amory JK, et al. Are physicians aware of which of their patients have indwelling urinary catheters? Am J Med 2000;109:476-80.

6 Klevens RM, Edwards JR, Richards CL Jr, et al. Estimating health care-associated infections and deaths in U.S. hospitals, 2002. Public Health Rep 2007;122:160-6.

7 Scott R. The direct medical costs of healthcare-associated infections in US hospitals and the benefits of prevention. March 2009. Publication No. CS200891-A. http://www.cdc. gov/hai/pdfs/hai/scott_costpaper.pdf; (accessed 10 May 2013).

8 Anderson DJ, Kirkland KB, Kaye KS, et al. Underresourced hospital infection control and prevention programs: penny wise, pound foolish?. Infect Control Hosp Epidemiol 2007;28:767-73.

9 Tambyah PA, Knasinski V, Maki DG. The direct costs of nosocomial catheter-associated urinary tract infection in the era of managed care. Infect Control Hosp Epidemiol 2002;23:27-31.

10 Greene MT, Chang R, Kuhn L, et al. Predictors of hospital-acquired urinary tract-related bloodstream infection. Infect Control Hosp Epidemiol 2012;33:1001-7.

11 Department of Health and Human Services, Centers for Medicare \& Medicaid Services. Medicare program; changes to the hospital inpatient prospective payment systems and fiscal year 2009 rates. Fed Regist 2008;73:48473-91.

12 Cardo D, Dennehy PH, Halverson P, et al. Moving toward elimination of healthcare-associated infections: a call to action. Am J Infect Control 2010;38:671-5.

13 Department of Health and Human Services (HHS). HHS Action Plan to Prevent Healthcare-Associated Infections, Appendix G 2009. http://www.hhs.gov/ash/initiatives/hai/ appendices.html\#appendix_g (accessed 8 Dec 2012).
14 Gould CV, Umscheid CA, Agarwal RK, et al. Guideline for prevention of catheter-associated urinary tract infections 2009. Infect Control Hosp Epidemiol 2010;31:319-26.

15 Hooton TM, Bradley SF, Cardenas DD, et al. Diagnosis, prevention, and treatment of catheter-associated urinary tract infection in adults: 2009 International Clinical Practice Guidelines from the Infectious Diseases Society of America. Clin Infect Dis 2010;50:625-63.

16 Lo E, Nicolle L, Classen D, et al. Strategies to prevent catheter-associated urinary tract infections in acute care hospitals. Infect Control Hosp Epidemiol 2008;29(Suppl 1):S41-50.

17 Greene L, Marx J, Oriola S. An APIC Guide to the elimination of catheter-associated urinary tract infections (CAUTIs): developing and applying facility-based prevention interventions in acute and long-term care settings 2008. http://www.apic.org/Resource_/ EliminationGuideForm/ c0790db8-2aca-4179-a7ae-676c27592de2/File/ APIC-CAUTI-Guide.pdf (accessed 8 Dec 2012).

18 Saint S. Chapter 15. Prevention of Nosocomial Urinary Tract Infections. Making Health Care Safer: A Critical Analysis of Patient Safety Practices. Evidence Report/Technology Assessment. Vol 43. AHRQ Publication No. 01-E058. Rockville, MD: Agency for Healthcare Research and Quality, 2001:149-62. http://www.ahrq.gov/clinic/ptsafety/pdf/chap15. pdf (accessed 8 Dec 2012).

19 Saint S, Olmsted RN, Fakih MG, et al. Translating health care-associated urinary tract infection prevention research into practice via the bladder bundle. Jt Comm J Qual Patient Saf 2009;35:449-55.

20 Fakih MG, Watson SR, Greene MT, et al. Reducing inappropriate urinary catheter use: a statewide effort. Arch Intern Med 2012;172:255-60.

21 Meddings J, Saint S. Disrupting the life cycle of the urinary catheter. Clin Infect Dis 2011;52:1291-3.

22 Meddings J, Rogers MA, Macy M, et al. Systematic review and meta-analysis: reminder systems to reduce catheter-associated urinary tract infections and urinary catheter use in hospitalized patients. Clin Infect Dis 2010;51:550-60.

23 Fakih MG, Pena ME, Shemes S, et al. Effect of establishing guidelines on appropriate urinary catheter placement. Acad Emerg Med 2010;17:337-40.

24 Knoll BM, Wright D, Ellingson L, et al. Reduction of inappropriate urinary catheter use at a Veterans Affairs hospital through a multifaceted quality improvement program. Clin Infect Dis 2011;52:1283-90.

25 Gardam MA, Amihod B, Orenstein P, et al. Overutilization of indwelling urinary catheters and the development of nosocomial urinary tract infections. Clin Perform Qual Health Care 1998;6:99-102.

26 Cornia PB, Amory JK, Fraser S, et al. Computer-based order entry decreases duration of indwelling urinary catheterization in hospitalized patients. Am J Med 2003;114:404-7.

27 Patrizzi K, Fasnacht A, Manno M. A collaborative, nurse-driven initiative to reduce hospital-acquired urinary tract infections. J Emerg Nurs 2009;35:536-9.

28 Andreessen L, Wilde MH, Herendeen P. Preventing catheter-associated urinary tract infections in acute care: the bundle approach. J Nurs Care Qual 2012;27:209-17.

29 Gokula RM, Smith MA, Hickner J. Emergency room staff education and use of a urinary catheter indication sheet improves appropriate use of foley catheters. Am J Infect Control 2007;35:589-93. 
30 Rothfeld AF, Stickley A. A program to limit urinary catheter use at an acute care hospital. Am J Infect Control 2010;38:568-71.

31 Topal J, Conklin S, Camp K, et al. Prevention of nosocomial catheter-associated urinary tract infections through computerized feedback to physicians and a nurse-directed protocol. Am J Med Qual 2005;20:121-6.

32 Stephan F, Sax H, Wachsmuth M, et al. Reduction of urinary tract infection and antibiotic use after surgery: a controlled, prospective, before-after intervention study. Clin Infect Dis 2006;42:1544-51.

33 Dumigan DG, Kohan CA, Reed CR, et al. Utilizing national nosocomial infection surveillance system data to improve urinary tract infection rates in three intensive-care units. Clin Perform Qual Health Care 1998;6:172-8.

34 Elpern EH, Killeen K, Ketchem A, et al. Reducing use of indwelling urinary catheters and associated urinary tract infections. Am J Crit Care 2009;18:535-41; quiz 542.

35 Fuchs MA, Sexton DJ, Thornlow DK, et al. Evaluation of an evidence-based, nurse-driven checklist to prevent hospital-acquired catheter-associated urinary tract infections in intensive care units. J Nurs Care Qual 2011;26:101-9.

36 Reilly L, Sullivan P, Ninni S, et al. Reducing foley catheter device days in an intensive care unit: using the evidence to change practice. AACN Adv Crit Care 2006;17:272-83.

37 Schultz P, Aljawawdeh A, Hopp T. EB105: Reducing use of indwelling urinary catheters with a nurse-driven protocol. Crit Care Nurse 2011;31:e42-e42. (Abstract).

38 van den Broek PJ, Wille JC, van Benthem BHB, et al. Urethral catheters: can we reduce use? BMC Urology 2011;11:10.

39 Lee YY, Tsay WL, Lou MF, et al. The effectiveness of implementing a bladder ultrasound programme in neurosurgical units. J Adv Nurs 2007;57:192-200.

40 Frederickson M, Neitzel JJ, Miller EH, et al. The implementation of bedside bladder ultrasound technology: effects on patient and cost postoperative outcomes in tertiary care. Orthop Nurs 2000;19:79-87.

41 Slappendel R, Weber EW. Non-invasive measurement of bladder volume as an indication for bladder catheterization after orthopaedic surgery and its effect on urinary tract infections. Eur J Anaesthesiol 1999;16:503-6.

42 Foxman B. Epidemiology of urinary tract infections: incidence, morbidity, and economic costs. Am J Med 2002;113(Suppl 1A):5S-13S.

43 Aaronson DS, Wu AK, Blaschko SD, et al. National incidence and impact of noninfectious urethral catheter related complications on the Surgical Care Improvement Project. J Urol 2011;185:1756-60.

44 Jain M, Miller L, Belt D, et al. Decline in ICU adverse events, nosocomial infections and cost through a quality improvement initiative focusing on teamwork and culture change. Qual Saf Health Care 2006;15:235-9.

45 Weitzel T. To cath or not to cath? Nursing 2008;38:20-1.

46 Titsworth WL, Hester J, Correia T, et al. Reduction of catheter-associated urinary tract infections among patients in a neurological intensive care unit: a single institution's success. J Neurosurg 2012;116:911-20.

47 Apisarnthanarak A, Thongphubeth K, Sirinvaravong S, et al. Effectiveness of multifaceted hospitalwide quality improvement programs featuring an intervention to remove unnecessary urinary catheters at a tertiary care center in Thailand. Infect Control Hosp Epidemiol 2007;28:791-8.
48 Crouzet J, Bertrand X, Venier AG, et al. Control of the duration of urinary catheterization: impact on catheter-associated urinary tract infection. J Hosp Infect 2007;67:253-7.

49 Huang WC, Wann SR, Lin SL, et al. Catheter-associated urinary tract infections in intensive care units can be reduced by prompting physicians to remove unnecessary catheters. Infect Control Hosp Epidemiol 2004;25:974-8.

50 Fakih MG, Dueweke C, Meisner S, et al. Effect of nurse-led multidisciplinary rounds on reducing the unnecessary use of urinary catheterization in hospitalized patients. Infect Control Hosp Epidemiol 2008;29:815-19.

51 Seguin P, Laviolle B, Isslame S, et al. Effectiveness of simple daily sensitization of physicians to the duration of central venous and urinary tract catheterization. Intensive Care Med 2010;36:1202-6.

52 Saint S, Kaufman SR, Thompson M, et al. A reminder reduces urinary catheterization in hospitalized patients. Jt Comm J Qual Patient Saf 2005;31:455-62.

53 Bruminhent J, Keegan M, Lakhani A, et al. Effectiveness of a simple intervention for prevention of catheter-associated urinary tract infections in a community teaching hospital. Am J Infect Control 2010;38:689-93.

54 Murphy D, Francis K, Litzenberger M, et al. Reducing urinary tract infection: a nurse-initiated program. Pa Nurse 2007;62:20.

55 Robinson S, Allen L, Barnes MR, et al. Development of an evidence-based protocol for reduction of indwelling urinary catheter usage. Medsurg Nurs 2007;16:157-61.

56 Gotelli JM, Merryman P, Carr C, et al. A quality improvement project to reduce the complications associated with indwelling urinary catheters. Urologic Nursing 2008;28:465-7.

57 Loeb M, Hunt D, O'Halloran K, et al. Stop orders to reduce inappropriate urinary catheterization in hospitalized patients: a randomized controlled trial. J Gen Intern Med 2008;23:816-20.

58 Voss AB. Incidence and duration of urinary catheters in hospitalized older adults: before and after implementing a geriatric protocol. J Gerontol Nurs 2009;35:35-41.

59 Wenger JE. Cultivating quality: reducing rates of catheter-associated urinary tract infection. Am J Nurs 2010;110:40-5.

60 Adams D, Bucior H, Day G, et al. HOUDINI: make that urinary catheter disappear-nurse-led protocol. J Infect Prev 2012;13:44-6.

61 Roser L, Altpeter T, Anderson D, et al. A nurse driven Foley catheter removal protocol proves clinically effective to reduce the incidents of catheter related urinary tract infections. Am J Infect Control 2012;40:e92-93 (Abstract).

62 Fakih MG, Rey JE, Pena ME, et al. Sustained reductions in urinary catheter use over 5 years: Bedside nurses view themselves responsible for evaluation of catheter necessity. Am J Infect Control 2013;41:236-9.

63 Pickard R, Lam T, Maclennan G, et al. Antimicrobial catheters for reduction of symptomatic urinary tract infection in adults requiring short-term catheterisation in hospital: a multicentre randomised controlled trial. Lancet 2012;380:1927-35.

64 Pronovost PJ, Berenholtz SM, Needham DM. Translating evidence into practice: a model for large scale knowledge translation. BMJ 2008;337:a1714.

65 Damschroder LJ, Banaszak-Holl J, Kowalski CP, et al. The role of the champion in infection prevention: results from a 
multisite qualitative study. Qual Saf Health Care 2009;18:434-40.

66 Saint S, Kowalski CP, Forman J, et al. A multicenter qualitative study on preventing hospital-acquired urinary tract infection in US hospitals. Infect Control Hosp Epidemiol 2008;29:333-41.

67 Saint S, Kowalski CP, Banaszak-Holl J, et al. How active resisters and organizational constipators affect health care-acquired infection prevention efforts. Jt Comm J Qual Patient Saf 2009;35:239-46.

68 Krein SL, Kowalski CP, Hofer TP, et al. Preventing hospital-acquired infections: a national survey of practices reported by U.S. hospitals in 2005 and 2009. J Gen Intern Med 2012;27:773-9.

69 Fink R, Gilmartin H, Richard A, et al. Indwelling urinary catheter management and catheter-associated urinary tract infection prevention practices in Nurses Improving Care for Healthsystem Elders hospitals. Am J Infect Control 2012;40:715-20.

70 Conway LJ, Pogorzelska M, Larson E, et al. Adoption of policies to prevent catheter-associated urinary tract infections in United States intensive care units. Am J Infect Control 2012;40:705-10.

71 Saint S, Kowalski CP, Banaszak-Holl J, et al. The importance of leadership in preventing healthcare-associated infection: results of a multisite qualitative study. Infect Control Hosp Epidemiol 2010;31:901-7.

72 Fakih MG, Todd Greene M, Kennedy EH, et al. Introducing a population-based outcome measure to evaluate the effect of interventions to reduce catheter-associated urinary tract infection. Am J Infect Control 2012;40:359-64.

73 Palumbo AJ, Loveless PA, Moll ME, et al. Evaluation of healthcare-associated infection surveillance in Pennsylvania hospitals. Infect Control Hosp Epidemiol 2012; 33:105-11.

74 Meddings J, Saint S, McMahon LF Jr. Hospital-acquired catheter-associated urinary tract infection: documentation and coding issues may reduce financial impact of Medicare's new payment policy. Infect Control Hosp Epidemiol 2010;31:627-33.

75 Centers for Disease Control and Prevention (CDC). Carbapenem-Resistant Enterobacteriaceae Containing New Delhi Metallo-Beta-Lactamase in Two Patients-Rhode Island. MMWR Morb Mortal Wkly Rep 2012;61:446-8.

76 Marchaim D, Chopra T, Bogan C, et al. The burden of multidrug-resistant organisms on tertiary hospitals posed by patients with recent stays in long-term acute care facilities. Am J Infect Control 2012;40:760-5. 